\title{
Stochastic Methods on Random Recursive Graphs Having Scale-free Properties
}

\author{
Xiaomin Wang, Bing Yao ${ }^{\mathrm{a}}$ \\ College of Mathematics and Statistics \\ Northwest Normal University \\ Lanzhou, 730070, CHINA \\ aemilbox:yybb918@163.com
}

\author{
Ming $\mathrm{Yao}^{\mathrm{b}}$ \\ Department of Information Process and Control Engineering \\ Lanzhou Petrochemical College of Vocational Technology \\ Lanzhou, 730060, CHINA \\ bemail: yybm918@163.com
}

\begin{abstract}
In this paper, we recommend a stochastic recursive graph, on the basis of which we reform a kind of graph called random recursive graphs. We investigate analytically or numerically the statistical characteristics of random recursive graphs. The obtained results reveal that the properties of random recursive graphs are particularly rich, it is simultaneously scalefree. Besides, we present three approaches to illustrate the random recursive graphs following the power law behavior. All obtained analytical predictions are successfully contrasted with extensive numerical simulations. We discuss the gap between the deterministic network and random network.
\end{abstract}

Keywords—scale-free graph;continuum theory;master equation; rate equation

\section{INTRODUCTION}

It was observed recently that the distributions of several quantities in various growing networks have a power-law form. This scaling behavior was discovered in World Wide Web, in neural and social networks, in nets of citations of scientific papers and so on [1]. In fact, the researching of complex networks has a long-standing history but only now we become feel ourselves as subjects interior of the world of developing complex networks and start appreciate purport of the developing network problems. Mostly, the attention was concentrated on the degree distribution between the different vertices of complex networks and on the degree distribution of the number of connections with a vertex. The latter quantity is obviously simpler to obtain than the former one but even for it, in the case of the networks with scaling law, no exact results are realized.

In the process of researching complex networks, while a lot of models have been introduced to describe scale-free networks in real daily life, most of them are stochastic models. However, a certain of network models recently have been introduced by Barabasi, Ravasz and Vicsek, and Jung and Kim are deterministic. Barabasi et al pointed out that the deterministic scale-free networks which describing a simple model produces scale-free behavior in a deterministic fashion [2]. Jung and Kim proposed a deterministic model for scalefree properties, whose degree distribution follows a power law with the exponent [3]. After their analysis, some scholars put forward that several classical and deterministic scale-free networks, such as deterministic scale-free web [2], recursive graphs [4], high dimensional apollonian networks and maximal planar scale-free Sierpinksi networks [5] etc. These networks decrease power law distribution on the basis of the theory was proposed in [6].

After the deterministic scale-free, one focus on considering the random networks, which press close to concrete complex systems in our daily life. The dynamical properties of the scale-free model can be addressed using various analytical approaches. The methods are the continuum theory, the master equation, the rate equation and the generating function. The continuum theorem focuses on the dynamical of vertex degrees [7]. Widely used are the master equation approach of Dorgovtsev, Mendes and Samukhin [8] and the rate equation approach to solve the growing random network model was introduced by Krapivsky, Render and Leyvarz [9]. Other researches are in [10-15].

Comellas [4] has proposed a family of deterministic networks based on the well-known $q$-clique. These graphs possess good topological properties observed in some real systems. However, their deterministic construction is not line with the randomness of many real-world systems. In this paper, we appoint a class of stochastic recursive graphs, in relation to which a novel graph, named random recursive graphs. The obtained graph is scale-free and it displays the general topological structures of real systems: heavy-tailed degree distribution. We also apply three methods to illustrate the degree distribution of the random recursive graphs obey a scaling law. The methods are the continuum theory, the master equation, and the rate equation. All theoretical prediction are successfully confirmed by numerical simulations. We discuss the phenomenon between deterministic networks and random networks and propose some problems for future discussion.

\section{RANDOM RECURSIVE GRAPHS AND ITS ITERATIVE ALGORITHM}

\section{A. Random Recursive Graphs}

We first construct a random recursive graph from the deterministic recursive graphs. Then we will establish a random recursive graph based on the proposed stochastic graphs. Analogous to the recursive graphs, the random recursive graphs also start with a complete graph $K_{q}$. At step 1 , we perform a complete graph of its existing subgraphs isomorphic to $q$-clique a new vertex and joining in Figure 1. Then in each of the subsequent generations, an existing subgraphs isomorphic to $q$-clique is chosen randomly for which forms $q$ small $q$-clique of it. From this process, we can easily 
construct the random recursive graphs. As in the construction of the deterministic version [4], the native clique $K_{q}$, at time step $t=0$ are also remained the original situation. Figure 1 shows a graph derived from the random recursive graphs. We can also find that the deterministic recursive graphs contain the random recursive graphs.

According to the construction of random recursive graphs, we introduce a general iterative algorithm generating the graphs, and denote the random recursive graphs after $t$ iterations by $N(t), t \geq 0$. Initially ( $t=0), N(0)$ is a complete graph $K_{q}$. At step $t=1$, we add a new vertex into the original complete graph which form a complete graph isomorphic to $q$-clique. Thus, we obtain the graph $N(1)$. For $t \geq 1, N(t)$ is obtained from $N(t-1)$. At step $t-1$, we select at random an existing subgraphs isomorphic to $q$-clique, then $N(t)$ is produced.

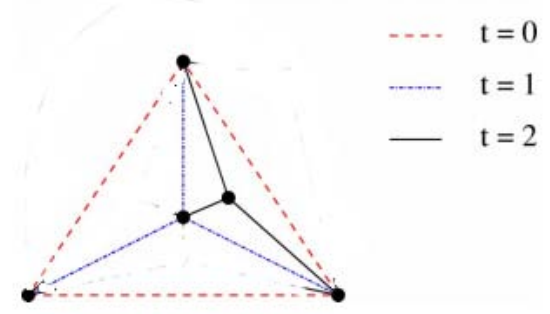

Figure-1 The sketch maps for the construction of random recursive graphs and its corresponding graph

Since at each time step, the numbers of vertices and edges increase by 1 and $q$, respectively, we can easily know that at step $t$, the graph consists of $N_{t}=t+q$ vertices and $E_{t}=t q+q(q+1) / 2$ edges. Thus, the relation $E_{t}=q N_{t}-q(q-1) / 2$ holds for all steps. Moreover, the recursive graphs are actually a deterministic tunable generalization of the scale-free growing networks introduced in [4] which one vertex is created per unit time and connects to both the ends of randomly chosen edge.

\section{ThreE APPROACHES FOR TESTIFYING POWER LAW DISTRIBUTION}

We now study the statistical properties of random recursive graphs, by applying three methods to validate the power degree distribution. The analytical approaches are completely deferent from those applied to the deterministic recursive graphs. Therefore, the considered graphs are pseudofractal graphs, which are similar to its deterministic version and some previously studied graphs.

\section{A. Continuum Theory}

First, we use the continuum theory to calculate the power law distribution of random recursive graphs. The continuum approach calculates the time dependence of degree $k_{i}$ of a given vertex $i$. This degree will increase every time a new vertex enters the system and links to vertex $i$, the probability of this process is $\Pi\left(k_{i}\right)$. Considering preferential attachment model the scale-free network, from the perspective of the vertex degree value change. We use the assumption that $k_{i}$ is a continuous real variable, the rate at which $k_{i}$ changes is proportional to $\Pi\left(k_{i}\right)$, According to [7], then we have the following dynamic equation

$$
\frac{\partial k_{i}}{\partial t}=q \prod\left(k_{i}\right)=q \frac{k_{i}}{\sum k_{j}}
$$

Every step we add $q$ edges, namely, totally adding 2tq edges, then we have $\sum k_{j}=2 q t+q(q+1)$ by the basic theorem of graph theory. Hence, $\frac{\partial k_{i}}{\partial t}=\frac{k_{i}}{2 t}$, and solve it with the initial condition that each vertex $i$ at its introduction has $k_{i}\left(t_{i}\right)=q$, the solution $k_{i}(t)=q\left(t / t_{i}\right)^{2}$ is combined with the distribution of $t_{i}$, so we can know the distribution $k_{i}$ that a vertex has a degree smaller than $k$ at any instant $t$, namely

$$
P\left(k_{i}(t)>k\right)=P\left(t_{i}>\frac{q^{2} t}{k^{2}}\right)
$$

At time step $t$, Barabasi et al [7] supposed that the values of $t_{i} \in[0, t]$ have a constant probability density $P\left(t_{i}\right)=1 /\left(q+t_{i}\right)$. Plugging this assumption into equation (2), we obtain

$$
P\left(t_{i}>\frac{q^{2} t}{k^{2}}\right)=1-\frac{q^{2} t}{k^{2}(t+q)}
$$

Furthermore,

$$
\begin{aligned}
P\left(k_{i}(t)<k\right) & =1-P\left(k_{i}(t) \geq k\right) \\
& =P\left(t_{i}>\frac{q^{2} t}{k^{2}}\right)=1-P\left(t_{i} \leq \frac{q^{2} t}{k^{2}}\right)
\end{aligned}
$$

Use the probability $P\left(t_{i}\right)=1 /\left(q+t_{i}\right)$ and equation (4), we can get the degree distribution

$$
\begin{aligned}
P(k) & =\frac{\partial P\left(k_{i}(t)<k\right)}{\partial k}=\frac{\partial}{\partial k}\left[1-\frac{q^{2} t}{k^{2}(t+q)}\right] \\
& =\frac{2 q^{2} t}{k^{3}(q+t)} \propto 2 q^{2} k^{-3}
\end{aligned}
$$

The equation (1) is a description at the view of average sense. According to $\Pi\left(k_{i}\right)$ distribution to choose the adding edge in real random process, it does not ensure that let each vertex degree increases like that and the scale-free and related models $[1,6,8,9]$ views networks as dynamical systems, assuming that they self-assemble and evolve in time through the addition and removal of vertices and edges. It is the so-called continuity method, so the actual random process needs to use other approaches described the evolution of distribution function, and it is not as an independent document.

\section{B. Master Equation}

This method was analytical introduced by Dorogovstev [8] We, now, use this method to explain the random recursive graphs. We label vertices by their birth times $t_{i}=0,1,2, \ldots, t$, and use $p\left(k, t_{i}, t\right)$ to denote the probability that at time $t$ a vertex 
created at time $t_{i}$ has its own degree $k$. The master equation growing the evolution of the degree distribution of individual vertex has the form

$$
\begin{aligned}
p\left(k, t_{i}, t+1\right) & =\left(1-\frac{k}{2 t q+q(q+1)}\right) p\left(k, t_{i}, t\right) \\
& +\frac{k}{2 t q+q(q+1)} p\left(k-1, t_{i}, t\right)+\delta_{q}
\end{aligned}
$$

With the initial condition $p\left(k, t_{i}, t_{i}\right)=\delta_{q}$. This accounts for two possibilities for a vertex first, with probability $k /(2 t q+q(q+1))$, it may get an extra edge from the new vertex while its existing edges remain undeleted; and second, with the complimentary probability $1-k /(2 t q+q(q+1))$ the vertex may remain in the former state with the former degree. It should be notes at equation (6) and all the following ones exact for all $t \geq 1$. The total degree distribution of the entire network can be obtained as

$$
P(k, t)=\frac{1}{t} \sum_{t_{i}=1}^{t} p\left(k, t_{i}, t\right)
$$

Using this and applying to $\sum_{t_{i}=0}^{t}$ both sides of equation (6), we get the following master equation for degree distribution

$$
\begin{aligned}
& (t+1) P(k, t+1)-p(k, t+1, t+1)=\left(1-\frac{k}{2 t q+q(q+1)}\right) P(k, t) \\
& +\frac{k}{2 t q+q(q+1)} P(k-1, t)+\delta_{q}
\end{aligned}
$$

Equation (6) implies that $P(k)$ is the solution of the recursive equation, value distribution is defined all vertices added to the system for all different times of the average behavior of vertex,

$$
P(k)=\lim _{t \rightarrow \infty} \frac{1}{t} \sum_{t_{i}} p\left(k, t_{i}, t+1\right)
$$

It can be obtained distribution formula from equation (6) and equation (7)

$$
P(k)=2 q(q+1) /[(k+q)(k+q+1)(k+q+2)] \sim 2 q^{2} k^{-3}
$$

This expression as $t \rightarrow \infty$ approaches the correspondence result of [1] in the frames of an approximate scheme, but the prefactors are different. In fact, the "mean field" approach used in [17], is equivalent to the continuous $k$ approximation in our discrete difference equations. Indeed, if we replace the finite difference with a derivative over $k$, we get the expression obtained in $[16,17]$.

\section{Rate Equation}

Rate equation describes another distribution space. Initially, there is only one $q$-clique in the graph. In the subsequent iterations, at each time step, a new vertex is created, so the total number of vertex is increased by 1 . Then at time step $t$, there are $(t q+1) q$-cliques. Note that, for an arbitrary given vertex, when it was born, it has a degree of $q$ clique and $(q+1)$-clique containing itself, and in the following steps, each of its two new neighbors separately generates $q$ clique involving it. So, the vertex which degree is $k$ form a clique at time step $t$, and the clique size denote $N_{k}(t)$, When there is new vertex enter the systems, $N_{k}(t)$ is likely to change, This happens with the probability $\frac{k N_{k}(t)}{\sum_{d} d N_{d}(t)}$, then the rate of change satisfies the following equation,

$$
\frac{d N_{k}}{d t}=\frac{(k-1) N_{k-1}(t)}{\sum_{d} N_{d}(t)}-\frac{k N_{k}(t)}{\sum_{d} N_{d}(t)}+\delta_{k, q}
$$

The first term on the right side of the equation (11) accounts for the process in which a vertex with $k-1$ link is connected to two new vertices, leading to a gain in the numbers of vertices with $k$ links. Since there are $N_{k-1}(t)$ vertices of degree $k-1$, such processes occur at a rate proportional to $(k-1) N_{k}(t)$, while the factor $t q+1$ converts this rate into a normalized probability. A corresponding role is played by the second term on the right side of equation (11). The last term on the right side of the equation (11) accounts for the continuous introduction of a new q clique with degree $q . N_{k}(t)$ similarly satisfy the equation $\sum_{k} k N_{k}(t)=2 t q+q(q+1)$. The first two moments are therefore independent of $\gamma$, while higher moments and the connectivity distribution itself do depend on $\gamma \cdot P(k)$ is the degree distribution. Substitute this relation into equation (11) to lead to the following recursive equation for infinite $t$. For linear connection, equation (11) can be solved for an arbitrary initial condition. We limit ourselves to the most interesting asymptotic regime $(t \rightarrow \infty)$ where the initial condition is irrelevant. We can obtain the recursive relation

$$
P(k)=(k-1) /(k+2) P(k-1)
$$

Solving for $P(k)$, in the long-time limit, we can obtain that

$$
P(k)=\lim _{t \rightarrow \infty} \frac{N_{k}(t)}{\sum_{d} d N_{d}(t)}
$$

Combining the equation (11) and equation (13) to lead to the following recursive equation for infinite $t$

$$
P(k)=2 q(q+1) / k(k+1)(k+2) \sim 2 q^{2} k^{-3}
$$

In the limit of large of $k, P(k) \sim 2 q^{2} k^{-3}$, which has the same degree exponent as BA-model and some hierarchical lattice models.

\section{DISCUSSION AND CONCLUSION}

In the above we list three approaches to testify the scaling law, and three methods applied in different ways to explain the power law degree distribution. The method can explain the network is scale-free graphs, because the degree distribution follows a power law distribution. Notice that when $t$ gets in large, the maximal degree of a vertex is roughly $k^{-3}$. First, we discuss the continuum theory, This method focus on the dynamics of vertices of degrees. The incompleteness of the information available to the local decision maker about the state of the full network, as well as the different interest driving the individual vertices, are the origin of the stochastic component in network evolution. Second, in the limit of long times, we have found that the exact solution of the master equation for the distribution of incoming links. This solution demonstrates the existence of the scaling region in a class of 
the growing networks with the preferential linking. Lastly, The rate equation approach to solve the growing random network model, a special case of which was accounted for the distribution of citations and other growing networks. This approach is ideally-suited for the growing random network and is much simpler than the standard probabilistic or generating function techniques. The rate equation formulation can be adapted to study more general evolving graph systems, such as networks with the site deletion and link re-arrangement. The method from different spatial angles to illustrate the graph is scale-free graphs.

Besides, we know the deterministic recursive graphs follow the power law $P_{\text {cum }}(k) \sim k^{1-\gamma}$ with $\gamma=1+\ln (q+1) / \ln q$. However, this family is infinite as $q$ can take any natural value starting from 2. Spaces between degrees of the spectrum grow with increasing $k$. Therefore, to relate the exponent of this discrete degree distribution to the standard $\gamma$ exponent of a continuous degree distribution for random scale-free networks, we use a cumulative distribution. The resultant recursive graphs have a power law distribution $P_{\text {cum }}(k) \sim k^{1-\gamma}$ with $\gamma=1+\ln (q+1) / \ln q$ displays scale-free behavior, with $2<\gamma<2.58496$. While in the random recursive graphs obey $P(k) \sim k^{-\gamma}$, with $\gamma=3$. In addition, The authors of [5] and [18] listed two network models, one is the deterministic Sierpinksi network model which degree distribution follow the scaling law $P_{\text {cum }}(k) \sim k^{1-\gamma}$ with $\gamma=2+\ln 2 / \ln 3$, while in randm Sierpinski network models, the degree distribution obeys the $P(k) \sim k^{-\gamma}$, with $\gamma=3$. Currently, we still not know what causes this phenomenon, and we guess that the randomness contribute to the results. We now propose some problems for further research.

1. We know the approaches of calcualtion are different. In the deterministic, most of schlors employed the method from the discrete, while in the random networks, in order to simplify the calcualtion by applying the continuous variable way to research network models. It may be the reason that contribute to $\gamma$ is different, that is, there is a large difference between them.

2. We command deterministic networks represent regular cases which are artifical systems, for example, recursive graphs, apollonian network, deterministic Sierpinksi networks and so on. On the other hands, random networks stand for irregular cases in some real systems, such as, BA model and random Sierpinksi networks. We are always aware that there exists a larger gap between regular and irregular cases. And how to reduce the gap is the key point of researching in future.

3. This idea also can be applied in other real fields to help us account for the appearance in real life. In the future, there exist some cases to research the network in the form of half deterministic and half random networks.

\section{ACKNOWLEDGMENT}

The authors greatfully thank the National Natural Science Foundation of China under grants No. 61163054 and No. 61363060.

\section{REFERENCES}

[1] Albert-Laszlo, Barabasi and Reka Albert. "Emergence of scaling in random networks”, Science, 286, , 509-512,(1999).

[2] Albert-Laszlo,Barabasi, Erzsebet Ravasz, Tamas Vicsek. "Deterministic scale-free networks”,Physica A299, 559-564, (2001).

[3] Jung S, Kim S, Kahng B. "Geometric fractal growth model for scale-free networks”, Physical Review E 65, (5), 056101-056106,2002.

[4] Francesc Comellas, Spain Guillaume Fertin, Andre Raspaud. "Recursive graphs with small-world scale-free properties”, Physical Review E 69, 037104, 2004.

[5] Zhongzhi zhang, Francesc Comellas, Guillname Fertin, lili Rong. High dimensional apollonian networks. Physical A, 2005. Zhongzhi zhang, Shuigeng Zhou, Lujun Fang, Jihong Guan, Yichao zhang. Maximal planar scale-free Sierpinski networks with small-world effect and power-law strength-degree correlation. Physical A, 2007, 380, 621.

[6] S.N.Dorogovtesv, A.V. Goltsev, J.F.F.Mendes. Pseudofractal sacle-free web. Physical Review E 65, 066122-066126.

[7] Albert-Laszlo, Barabasi, Zoltan Dezso, Erzsebet Ravasz, Soon-Hyung Yook and Zoltan Oltvai. Scale-free and hierarchical structures in complex networks. MODELING OF COMPLEX SYSTEMS: Seventh Granada Lectures (2002). AIP Conference Proceedings, Volume 661,(2003), 1-16.

[8] S. N. Dorogovtsev, J. F. F. Mendes and A. N. Samukhin. Structure of Growing Networks with Preferential Linking. Physical Review Letters, 2000, 85, 21, 4633-4636.

[9] P. L.Krapivsky, S. Render and F. Leyvarz. Connectivity of growing random networks. Physical Review Letters, 2000, 85, 21, 4629-4632.

[10] M. E. J Newman, S.H. Strogatz, D.J. Watts. Random graphs with arbitrary degree distributions and their applications. Physical Review Letters, 2001, 64, 2, 026118, 1-17.

[11] Xia Liu, Bing Yao, Wanjia Zhang, Xiang-en Chen, Xinsheng Liu, Ming Yao.The 2014 International Conference on Information and Communication Technologies (ICT2014) will be held 16th-18th May 2014 in Nanjing, China.2014 pp35-38. Bing Yao, Ming Yao,Xiang-en Chen,Xia Liu, Wanjia Zhang 2013,513-517,65-68.

[12] Xiaomin Wang, Xiyang Zhao, Bing Yao, Ming Yao, Xiang-en Chen. Engineering solutions for industrial production, Volumes 775, 2015, 431-435.

[13] Bing Yao, Xia Liu, Wanjia Zhang, Xiang-en Chen, Ming Yao, Xuezhao,Zheng. 2013 International Conference on High Performance Computing and Communications, (IEEE2013) Volumes 380-384,36803684. Bing Yao, Hongyu Wang, Ming Yao, Xiang'en Chen, Chao Yang, and Xiaomin Zhang. Third International Conference on Information Science and Technology March 23-25, 2013; 738-743., Jiangsu, China. IEEE 978-1-4673-2764-0/13. Bing Yao, Zhong-fu Zhang and Jian-fang Wang. Acta Mathematicae Applicatae Sinica, English Series, 2010, 26(4), 607-616. Bing Yao, Xia Liu, Wan-jia Zhang, Xiang-en Chen, Xiao-min Zhang, Ming Yao, Zheng-xue Zhao. Applying Graph Theory To The Internet of Things. 2013 IEEE International Conference on High Performance Computing and Communications and 2013 IEEE International Conference on Embedded and Ubiquitous Computing, 2354-2361.

[14] E.R.Colman, G.J. Rodgers. Complex scale-free networks with tunable power-law exponent and clustering. Phyical A, 2013, 392, 5501-5510.

[15] Linjun Zhang, Michael Small, Kevin Judd. Exactly scale-free scale-free networks. Physical A 2015, 433, 182-197.

[16] Albert-Laszlo, Barabasi, Eric Bonabeau., Scale-free networks. Scientific American, 2003, 288, 60-69.

[17] R.Albert, H.Jeong. Physica A 272, 173, 1999.

[18] Zhang Zhonhzhi, Zhou Shuigeng, Su zhan, Zou tao, Guan jihong. Random Sierpinski networks with sacle-free small-world and modular $\begin{array}{lllll}\text { structure. } & \text { Physical }\end{array}$ 